\title{
A Research on Home Automation for Elderly and Physically Challenged People by using IOT
}

\author{
Chaitra N, Ashwini C S, Shyma Zaidi
}

\begin{abstract}
In this paper the home automation systems to support elderly and physically challenged people in providing them secure, safe and controlled environment is reviewed. Various technologies like Brain Controlled Interface (BCI) technology, Human Computer Interface system, GSM SMS services, Bluetooth, PIC Microcontroller with ZigBee modulation, Voice controlled home automation, Novel Electroculogram (EOG), Assistive Visible Light Communication $(V L C)$ etc. are discussed. By using smart home technology, the life of such people becomes easier and comfortable. The proposed paper is dedicated to old age and physically impaired people.

Index Terms - Home Automation, IOT, Elderly and Physically Challenged.
\end{abstract}

\section{INTRODUCTION}

Home automation systems are going to be very common in the near future. The block diagram of a typical home automation system which consists of sensors, appliances and connecting devices which can be controlled remotely is shown in Fig 1. Standard communication protocols are used for their communication between such systems [1]. For example, old age people or people with disabilities can control the various appliances around the home. They can switch on/ off the devices without any assistance [2].

IoT can be defined as inter-connection of physical devices, buildings and integrated with electronics, software, sensors through internet connectivity [3]. IoT based home automation will help the user to use a home appliance by connecting the devices to the internet. The modern homes are automated through the internet. Here home appliances are to control by using various wireless devices like laptops, tablets and smartphones. All these appliances and system contain sensors that connects them to a network. This is where IoT comes into place and makes it such an integral part of home automation.

These days all homes are interfaced by various electronic devices - Everything from a Television to CCTV. When these devices are connected with various software and the internet connection such devices become IoT. Home automation using IoT has become extremely helpful for physically impaired people in various ways like smart

Revised Manuscript Received on April 12, 2019.

Chaitra N,Department of Electronics and Communication Engineering, Vidyavardhaka College of Engineering, Mysuru, Karnataka 570002.India.(E-mail: chaitra5448@gmail.com)

Ashwini C S,Department of Electronics and Communication Engineering, Vidyavardhaka College of Engineering, Mysuru, Karnataka 570002. India (E-mail: ashwinichandramma@gmail.com)

ShymaZaidi, Department of Electronics and Communication Engineering, Vidyavardhaka College of Engineering, Mysuru, Karnataka 570002. India (E-mail: shymazaidi@ vvce.ac.in) lightning, and energy application. One can control all functions remotely in and around the home. The system can also perform face recognition within a camera itself because of which security, automation and IoT have become areas of amazing innovation.

\section{LITERATURE REVIEW}

Rizvi et al. [1], discussed the power management systems for appliances in their paper. The main aim is to make life more comfortable for elderly and handicapped people. The elderly and impaired people can make use of this technology. The appliances are operated by sending message through Android application and Bluetooth technology can also be used. The developed system helps the user to remotely control home appliances. GSM Network technology helps the user to control their appliances from any part of the world. Whereas Bluetooth network enables the user to control devices within specific range. Further the system can be improved by interfacing with sensors.

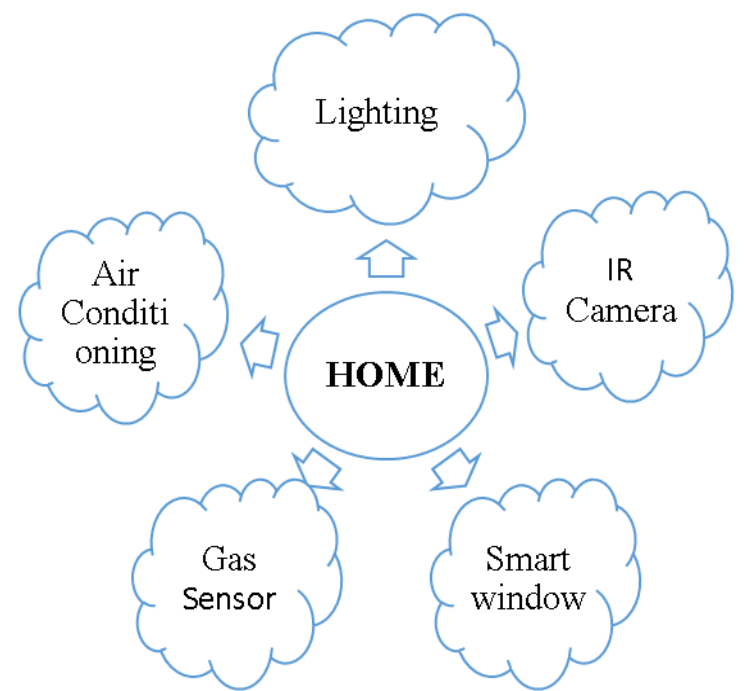

Fig. 1: Block Diagram of Typical Home Automation System

In the paper [2], the main objective is to build smart home which is cheaper when compared to all other home automation technologies. The interfaced system so simple as possible so that it makes physically impaired people more efficiently use it. Here the system can be operated using google assistant and android application. This system is

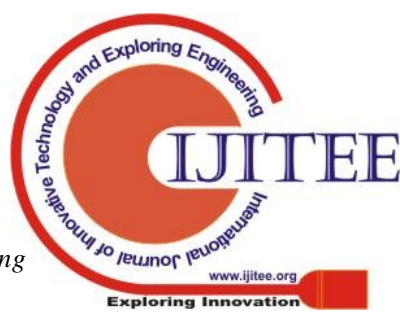


implemented by using Node MCU ESP8266 and WIFI mode of transmission. Fire base database is used to store real time data. The implemented system is flexible to work with number of devices that could be controlled from any part of the world.

A smart home automation has been developed using techniques like Python, OpenCV, Raspberry pi etc. particularly for old aged people in paper [3]. A door monitoring system is developed by detecting and recognizing the face of the user by camera which will be installed outside the door. Here, regulator of a fan and light can be switched on/off using the user voice command. Moreover, the system which is implemented using raspberry pi module are controlled or operated using android application which are controlled using voice. The proposed system is reasonably cheap and easy to run.

Rathnayake et al. [4] discuss the HIC (Human Computer Interaction) technology which reduces the gap between the human and computer. The system is an aid for physically challenged including people with speaking and hearing disabilities. The home appliances are controlled through voice and gesture commands which are sensed by Kinect motion sensor. In this paper voice and gesture recognition are one of the special consideration and HCI technology is the easiest way of communicating or interacting with hardware systems.

In the paper [5], home automation system supports mainly for elderly and physically disabled people. In this paper the home automation controls all lights and electrical appliances through the voice command. Here the system is interfaced through Arduino microcontroller for connecting appliances and Bluetooth module for signal transfer. The entire system here uses one of the larger and real database communication and thus enhance the whole system and also Bluetooth module can be replaced with specific component which may increase the range of accessibility.

In the paper [6], the implemented model is very useful for old aged and handicapped people. Home automation and its security can be achieved by using technologies like GSM, Bluetooth, PIC microcontroller with ZigBee and IoT etc. Smart home system provides security and reduces stress and it also makes the life of old aged and handicapped people simple. Home automation is very easy to use and main aim is to save electricity.

In the paper [7], Brain controlled Interface is a communication pathway between wired brain and external device. This technology helps to convert signal into action by collecting waves from different location of human brain. When this technology is combined with the home automation it helps for physically disabled people. In future scenario most of the applications can be controlled by implementing this technique. This technology can be further improved by making detailed studies on signals that comes from brain. Here the main aim was to control many devices without causing any motion which would improve the standard of living.

In the paper [8], the main aim of an assistive VLC technology is to improve the quality of life for disabled and elderly people. Here, a novel electro-occulogram (EOG) signal based assistive visible light communication (VLC) is used to implement a smart home. The system is operated by capturing the eye movement using an electrode $(\mathrm{AgCl})$. The user eye movement patterns were transmitted to the smart devices through the VLC link. This system controls many devices by producing eye movement patterns. This system is more accurate this can be used on practical VLC technology in smart home and health sectors.

In the paper [9], the objective of this paper is to make the physically impaired or elderly people more self-dependent. This intelligent based system operates on voice commands. This implemented system consists of voice recognition and processing technology and wireless communication. This system is cost effective and efficient and it is also easy to implement. The proposed model works effectively with coverage up to 10 meters. The proposed system has some of the disadvantages like people with speech impaired cannot use this system and the system is not highly noise resistant.

In the paper [10], the main aim of this paper is to mainly concentrate on physically disabled and paralyzed people. The (BCI) Brain Computer Interface is one of the technology where transmission takes place between human brain and external device. The ARM7 processor is used as interfacing device. This paper represents the design and implementation of BCI technique were EEG based brain signals are used to operate or control home appliances. Here it consists of Neurosky brainwave sensor with a dry electrode. The proposed system is implemented for controlling physical devices using brain signals. This system is useful in industries and hospitality.

\section{DISCUSSIONS \& RESULTS}

From all the above papers, every smart home system uses different technologies where Android phone plays a very important role in all the types of system [3]. The Brain computer interface system uses the brain signals to control appliances [7]. GSM network system will enable the user to control the things from all around the world whereas Bluetooth network system will be able to control within specific range [1]. In Wi-Fi based home automation system can be controlled by using Wi-Fi mode of transmission [2].

Advantages:

Cost effective

Greater comfort and convenience

User friendly

\section{CONCLUSION}

From all the above paper we can conclude that, the smart home automation can be developed by using various technologies. The technologies which is discussed above has both advantages and disadvantages. Here the main objective is to make the elderly and physically challenged population more self-dependent. Smart home system reduces stress by ensuring you to provide security to your home. In future scenario artificial Intelligence can also be included for further enhancement of this field. 


\section{ACKNOWLEDGEMENT}

The authors express gratitude towards the assistance provided by Accendere Knowledge Management Services Pvt Ltd for the expert advice.

\section{REFERENCES}

1. S. Rizvi, I. Sohail, M. M. Saleem, A. Irtaza, M. Zafar, and M. Syed, "A Smart Home Appliances Power Management System for Handicapped, Elder and Blind People," in 2018 4th International Conference on Computer and Information Sciences (ICCOINS), 2018, pp. 1-4.

2. H. V. Bhatnagar, P. Kumar, S. Rawat, and T. Choudhury, "Implementation model of Wi-Fi based Smart Home System," in 2018 International Conference on Advances in Computing and Communication Engineering (ICACCE), 2018, pp. 2328.

3. V. D. Vaidya and P. Vishwakarma, "A Comparative Analysis on Smart Home System to Control, Monitor and Secure Home, based on technologies like GSM, IOT, Bluetooth and PIC Microcontroller with ZigBee Modulation," in 2018 International Conference on Smart City and Emerging Technology (ICSCET), 2018, pp. 1-4.

4. K. A. S. V. Rathnayake, W. K. I. L. Wanniarachchi, and W. H. K. P. Nanavakkara, "Human Computer Interaction System for Impaired People by using Kinect Motion Sensor: Voice and Gesture Integrated Smart Home," in 2018 Second International Conference on Inventive Communication and Computational Technologies (ICICCT), 2018, pp. 531-536.

5. S. Mary, S. Benigna, H. Devi, S. Ravichandran, R. Angeline, and B. Tech, "Voice Controlled Home Automation System," J. Netw. Commun. Emerg. Technol., vol. 8, 2018.

6. B. Vaidya, A. Patel, A. Panchal, R. Mehta, K. Mehta, and P. Vaghasiya, "Smart home automation with a unique door monitoring system for old age people using Python, OpenCV, Android and Raspberry pi," in 2017 International Conference on Intelligent Computing and Control Systems (ICICCS), 2017, pp. 82-86.

7. A. A. Ghodake and S. D. Shelke, "Brain controlled home automation system," in 2016 10th International Conference on Intelligent Systems and Control (ISCO), 2016, pp. 1-4.

8. D. R. Dhatchayeny, W. A. Cahyadi, and Y.-H. Chung, "An Assistive VLC Technology for Smart Home Devices Using EOG," Wirel. Pers. Commun., vol. 98, no. 1, pp. 81-89, Jan. 2018.

9. R. Hajare, M. Gowda, S. Jain, P. Rudraraju, and A. Bhat, "Design and development of voice activated intelligent system for elderly and physically challenged," in 2016 International Conference on Electrical, Electronics, Communication, Computer and Optimization Techniques (ICEECCOT), 2016, pp. 372-376.

10. P. Adale, "A Brain Computer Interface for Smart Home Control,” Asian J. Converg. Technol., Mar. 2018. 